\title{
On the Convexity of Independent Set Games*
}

\author{
Han Xiaoł Yuanxi Wang, Qizhi Fang \\ School of Mathematical Sciences \\ Ocean University of China \\ Qingdao, China
}

\begin{abstract}
Independent set games are cooperative games defined on graphs, where players are edges and the value of a coalition is the maximum cardinality of independent sets in the subgraph defined by the coalition. In this paper, we investigate the convexity of independent set games, as convex games possess many nice properties both economically and computationally. For independent set games introduced by Deng et al. [5], we provide a necessary and sufficient characterization for the convexity, i.e., every non-pendant edge is incident to a pendant edge in the underlying graph. Our characterization immediately yields a polynomial time algorithm for recognizing convex instances of independent set games. Besides, we introduce a new class of independent set games and provide an efficient characterization for the convexity.
\end{abstract}

Keywords: Cooperative game theory, independent set, convexity.

Mathematics Subject Classification: 05C57, 91A12, 91A43, 91A46.

\section{Introduction}

Cooperative games form an important class in game theory, which have a lot of applications in economics, computer science and mathematics. One major problem in cooperative games is to maximize the profit by cooperation and allocate the profit among participants in the cooperation. There are many criteria for evaluating how "good" an allocation is, such as fairness and stability. Emphases on different criteria lead to different solution concepts, e.g., the core, the Shapley value, the nucleolus, the bargaining set, and the von Neumann-Morgenstern solution [19]. Among those solution concepts, the core which addresses the issue of stability is one of the most attractive solution concepts. The core is the set of allocations where no coalition has an incentive to split

${ }^{*}$ This work was supported in part by National Natural Science Foundation of China $(11871442,11971447)$ and Fundamental Research Funds for the Central Universities (201713051, 201964006).

${ }^{\dagger}$ Corresponding author. Email: hxiao@ouc.edu.cn. 
off from the grand coalition, and does better on its own. Hence a game with a non-empty core is especially interesting. A special subclass of games with a non-empty core is formed by convex 1 games.

Convex games were introduced by Shapley [18, which exhibit many desirable properties in cooperative game theory. In particular, (i) the core is always non-empty and a core allocation can be found in polynomial time [18; ;ii) testing whether an allocation belongs to the core can be performed in polynomial time [6]; (iii) computing the nucleolus can be done in polynomial time [12]; (iv) there is an appealing snowball effect, i.e., the incentive to join a coalition increases as the coalition grows larger [18. We refer to [14, 18, for many interesting properties of convex games involving other solution concepts. Hence the convexity of cooperative games has attracted a lot of research efforts, especially for cooperative games arising from combinatorial optimization problems. However, only a few combinatorial optimization games are universally convex [2, 3, 8, 13]. Hence one working direction is to characterize the condition for convexity. There is a line of research where convexity/concavity of cooperative games is characterized by the property of underlying graphs. Van den Nouweland and Borm [20] showed that communication vertex games are convex if and only if the underlying graph is cycle-complete and communication arc games are convex if and only if the underlying graph is cycle-free. Herer and Penn [9] showed that Steiner traveling salesman games are concave if the underlying graph is a 1-sum of $K_{4}$ and outerplanar graphs. Hamers [7] showed that Chinese postman games are concave if the underlying graph is weakly cyclic. Okamoto [15] showed that vertex cover games are concave if and only if the underlying graph is $\left(K_{3}, P_{4}\right)$-free, and coloring games are concave if and only if the underlying graph is complete multipartite. Based on the result of Hamers [7], Albizuri and Hamers [1] characterized the concavity of some variants of Chinese postman games. Kobayashi and Okamoto [10] initialized the study for the concavity of spanning tree games, where a sufficient condition and a necessary condition were given separately. Koh and Sanità [11] provided the first necessary and sufficient characterization for the concavity of spanning tree games. Platz [16] gave a complete characterization for the concavity of multi-depot Steiner traveling salesman games.

In this paper, we focus on the convexity of independent set games. Independent set games were introduced by Deng, Ibaraki and Nagamochi [5], which model the following scenario with projects and participants. Every participant is suitable for two projects but only allowed to join one project. Every project requires all suitable participants to cooperate to be done. The problem of maximizing doable projects can be viewed as a maximum independent set problem where projects are vertices and every participant is an edge joining two suitable projects. We provide a

\footnotetext{
${ }^{1}$ concave for cooperative games minimizing the cost by cooperation and allocating the cost among participants in the cooperation.
} 
necessary and sufficient characterization for the convexity of independent set games via the underlying graph. Our characterization implies that convex independent set games can be recognized effciently. Besides, we introduce a new class of independent set games and provide a necessary and sufficent characterization for the convexity.

The rest of this paper is structured as follows. In Section 2, notions and notations in graph theory and game theory are reviewed. Section 3 is devoted to an efficient characterization for the convexity of independent set games. In Section 4, we introduce a new class of independent set games and characterize the convexity. Section 5 concludes the results in this paper and discusses the directions of future work.

\section{Preliminaries}

\subsection{Graphs}

We assume that the readers have a moderate familiarity with graphs. However, some notions and notations used in this paper should be clarified before proceeding. Throughout, a graph is always finite, undirected and simple. For $n \in \mathbb{N}$, we use $K_{n}$ to denote the complete graph with $n$ vertices, use $K_{1, n}$ to denote the graph which is a star, i.e., a complete bipartite graph where one part has one vertex and the other part has $n$ vertices, use $C_{n}$ to denote the graph which is a cycle with $n$ vertices, and use $P_{n}$ to denote the graph which is a path with $n$ vertices. Since $K_{2}$ is isomorphic to $K_{1,1}$ and $P_{3}$ is isomorphic to $K_{1,2}$, both $K_{2}$ and $P_{3}$ are stars. Let $H$ be a graph. We use $V(H)$ to denote the vertex set of $H$ and use $E(H)$ to denote the edge set of $H$. A graph is said $H$-free if it contains no subgraph isomorphic to $H$. Let $G=(V, E)$ be a graph. For any $v \in V, N_{G}(v)$ denotes the set of vertices adjacent to $v, \delta_{G}(v)$ denotes the set of edges incident to $v$, and $d_{G}(v)$ denotes the degree of $v$. A vertex is isolated if it is a vertex with degree zero. A vertex is pendant if it is a vertex with degree one. An edge is pendant if it is incident to a pendant vertex. For any $U \subseteq V, G[U]$ denotes the induced subgraph of $G$. In particular, $G[\emptyset]$ is an empty graph which has no vertex. For any $F \subseteq E, V\langle F\rangle$ denotes the set of vertices incident only to edges in $F$, and $G[F]$ denotes the edge-induced subgraph of $G$. An independent set of $G$ is a vertex set $U \subseteq V$ such that $G[U]$ has no edge. An edge cover of $G$ is an edge set $F \subseteq E$ such that every vertex of $G$ is incident to an edge in $F$. For simplicity, we use $\alpha(G)$ to denote the maximum cardinality of independent sets in $G$.

\subsection{Cooperative games}

Let $\Gamma=(N, \gamma)$ be a cooperative game, where $N$ is the set of players and $\gamma: 2^{N} \rightarrow \mathbb{R}$ is the characterizatic function with $\gamma(\emptyset)=0$. A subset $S$ of $N$ is called a coalition and $N$ is called the

grand coalition. For each coalition $S, \gamma(S)$ represents the value distributed among the players in 
$S$. We call $\Gamma$ convex if for any $S, T \subseteq N$,

$$
\gamma(S)+\gamma(T) \leq \gamma(S \cap T)+\gamma(S \cup T)
$$

or equivalently, for any $i \in N$ and any $S \subseteq T \subseteq N \backslash\{i\}$,

$$
\gamma(S \cup\{i\})-\gamma(S) \leq \gamma(T \cup\{i\})-\gamma(T)
$$

We call $\Gamma$ concave if the reverse inequality holds in (1) or (2), and additive if the equality holds in (1) or (2).

An allocation of $\Gamma$ is a vector $\boldsymbol{x} \in \mathbb{R}^{N}$ which consists of proposed amounts to be shared by players in $N$. An allocation $\boldsymbol{x}$ is called efficient if $\sum_{i \in N} x_{i}=\gamma(N)$, and called coalitionally rational if $\sum_{i \in S} x_{i} \geq \gamma(S)$ for any $S \subseteq N$. The core of $\Gamma$ is the set of allocations that are efficient and coalitionally rational. The core of a cooperative game may be empty. A cooperative game is called balanced if the core is non-empty. Balanced games contain convex games as a special subclass [18].

\section{Convexity of independent set games}

The independent set game introduced by Deng et al. [5] is a cooperative game $\Gamma_{G}=(E, \gamma)$ defined on a graph $G=(V, E)$, where $E$ is the set of players and $\gamma: 2^{E} \rightarrow \mathbb{N}$ is the characteristic function such that $\gamma(F)=\alpha(G[V\langle F\rangle])$ for any $F \subseteq E$. We always assume that the underlying graph of an independent set game has no isolated vertex. Deng et al. [5] showed that an independent set game is balanced if and only if the underlying graph is a König-Egerváry graph [4, 17], where the maximum cardinality of independent sets is equal to the minimum cardinality of edge covers. In this paper, we show that an independent set game is convex if and only if every non-pendant edge is incident to a pendant edge in the underlying graph. We remark that (non-)pendant vertices and edges always refer to vertices and edges in the ground graph throughout this section.

Let $G=(V, E)$ be a graph without isolated vertices. Let $e \in E$ be an edge with endpoints $u_{1}, u_{2}$ and $F \subseteq E \backslash\{e\}$ be a set of edges. Clearly, neither $u_{1}$ nor $u_{2}$ belongs to $V\langle F\rangle$. Let $F^{\prime}=F \cup\{e\}$. It follows that $V\langle F\rangle \subseteq V\left\langle F^{\prime}\right\rangle$. Thus every independent set of $G[V\langle F\rangle]$ is also an independent set of $G\left[V\left\langle F^{\prime}\right\rangle\right]$. Notice that $V\left\langle F^{\prime}\right\rangle \subseteq V\langle F\rangle \cup\left\{u_{1}, u_{2}\right\}$. It follows that a maximum independent set of $G\left[V\left\langle F^{\prime}\right\rangle\right]$ is also a maximum independent set of $G[V\langle F\rangle]$ if it is a subset of $V\langle F\rangle$. Let $\Gamma_{G}$ be the independent set game on $G$. It follows that $\gamma\left(F^{\prime}\right) \geq \gamma(F)$. In the following, we distinguish two cases of $e$.

Lemma 1. If e is a pendant edge in $G$, then $\gamma\left(F^{\prime}\right)=\gamma(F)+1$. 
Proof. Let $I_{F}$ and $I_{F^{\prime}}$ be a maximum independent set in $G[V\langle F\rangle]$ and $G\left[V\left\langle F^{\prime}\right\rangle\right]$, respectively. We show that $\left|I_{F^{\prime}}\right|=\left|I_{F}\right|+1$. We may assume that $e$ is only incident to one pendant vertex, say $u_{1}$, since otherwise $\left|I_{F^{\prime}}\right|=\left|I_{F}\right|+1$ is trivial. It follows that $u_{1} \in V\left\langle F^{\prime}\right\rangle \backslash V\langle F\rangle$.

We claim that $u_{1} \in I_{F^{\prime}}$. To see this, we distinguish two cases of $V\left\langle F^{\prime}\right\rangle$. It is trivial if $V\left\langle F^{\prime}\right\rangle=$ $V\langle F\rangle \cup\left\{u_{1}\right\}$, as in this case $u_{1}$ is an isolated vertex in $G\left[V\left\langle F^{\prime}\right\rangle\right]$. Hence assume that $V\left\langle F^{\prime}\right\rangle=$ $V\langle F\rangle \cup\left\{u_{1}, u_{2}\right\}$. In this case we have $I_{F^{\prime}} \cap\left\{u_{1}, u_{2}\right\} \neq \emptyset$, since otherwise $I_{F^{\prime}} \cup\left\{u_{1}\right\}$ is an independent set in $G\left[V\left\langle F^{\prime}\right\rangle\right]$, which contradicts the maximality of $I_{F^{\prime}}$. Since $u_{1}$ is a pendant vertex, we may always assume that $u_{1} \in I_{F^{\prime}}$ by replacing $u_{2}$ with $u_{1}$ in $I_{F^{\prime}}$ when necessary.

Now on one hand, $I_{F^{\prime}} \backslash\left\{u_{1}\right\}$ is an independent set in $G[V\langle F\rangle]$. Thus $\left|I_{F^{\prime}} \backslash\left\{u_{1}\right\}\right|=\left|I_{F^{\prime}}\right|-1 \leq\left|I_{F}\right|$. On the other hand, since $u_{1}$ is a pendant vertex, $I_{F} \cup\left\{u_{1}\right\}$ is an independent set in $G\left[V\left\langle F^{\prime}\right\rangle\right]$. Thus $\left|I_{F^{\prime}}\right| \geq\left|I_{F} \cup\left\{u_{1}\right\}\right|=\left|I_{F}\right|+1$. It follows that $\left|I_{F^{\prime}}\right|=\left|I_{F}\right|+1$.

Lemma 2. If e is not a pendant edge in $G$, then $\gamma\left(F^{\prime}\right)=\gamma(F)$ or $\gamma\left(F^{\prime}\right)=\gamma(F)+1$. Moreover, if $\gamma\left(F^{\prime}\right)=\gamma(F)+1$, then every maximum independent set in $G\left[V\left\langle F^{\prime}\right\rangle\right]$ contains an endpoint of $e$ which is not adjacent to any pendant vertex.

Proof. Assume that $e$ is not a pendant edge in $G$. It is trivial that $\gamma(F) \leq \gamma\left(F^{\prime}\right)$. We show that $\gamma\left(F^{\prime}\right) \leq \gamma(F)+1$. Without loss of generality, assume that $V\left\langle F^{\prime}\right\rangle \neq V\langle F\rangle$, since otherwise we have $\gamma\left(F^{\prime}\right)=\gamma(F)$. Let $I_{F}$ and $I_{F^{\prime}}$ be a maximum independent set in $G[V\langle F\rangle]$ and $G\left[V\left\langle F^{\prime}\right\rangle\right]$, respectively. It suffices to show that $\left|I_{F^{\prime}}\right| \leq\left|I_{F}\right|+1$. Notice that $\left|I_{F^{\prime}}\right|=\left|I_{F}\right|$ if $I_{F^{\prime}} \cap\left\{u_{1}, u_{2}\right\}=\emptyset$, as $I_{F^{\prime}}$ is also a maximum independent set in $G[V\langle F\rangle]$. Hence, without loss of generality, assume that $u_{1} \in I_{F^{\prime}}$. Notice that $I_{F^{\prime}} \backslash\left\{u_{1}\right\}$ is an independent set in $G[V\langle F\rangle]$. It follows that $\left|I_{F}\right| \geq$ $\left|I_{F^{\prime}} \backslash\left\{u_{1}\right\}\right|=\left|I_{F^{\prime}}\right|-1$. Therefore, we have $\gamma(F) \leq \gamma\left(F^{\prime}\right) \leq \gamma(F)+1$.

Now further assume $\gamma\left(F^{\prime}\right)=\gamma(F)+1$. Recall that $u_{1} \in I_{F^{\prime}}$ is the endpoint of $e$. We show that $u_{1}$ is not adjacent to any pendant vertex by proving that $d_{G}(v) \geq 2$ for any $v \in N_{G}\left(u_{1}\right)$. Assume to the contrary that there is a vertex $v^{*} \in N_{G}\left(u_{1}\right)$ with $d_{G}\left(v^{*}\right)=1$. Then $\left\{u_{1}, v^{*}\right\}$ is a pendant edge, implying that $v^{*} \neq u_{2}$. It follows that $\delta_{G}\left(u_{1}\right) \subseteq F^{\prime}$ and $\delta_{G}\left(u_{1}\right) \backslash\{e\} \subseteq F$. Thus $\left\{u_{1}, v^{*}\right\}$ is an edge in $F$. Since $v^{*}$ is a pendant vertex, we have $v^{*} \in V\langle F\rangle$ and $u_{1} \notin V\langle F\rangle$, implying that $v^{*}$ is an isolated vertex in $G[V\langle F\rangle]$. Let $I_{F^{\prime}}^{\prime}=I_{F^{\prime}} \backslash\left\{u_{1}\right\}$. Notice that $I_{F^{\prime}}^{\prime}$ is an independent set in $G[V\langle F\rangle]$ with cardinality $\left|I_{F^{\prime}}\right|-1$. Since $v^{*}$ and $u_{1}$ are adjacent in $G\left[V\left\langle F^{\prime}\right\rangle\right]$, we have $v^{*} \notin I_{F^{\prime}}$. Then $I_{F^{\prime}}^{\prime} \cup\left\{v^{*}\right\}$ is an independent set in $G[V\langle F\rangle]$ with cardinality $\left|I_{F^{\prime}}\right|$, which contradicts $\gamma\left(F^{\prime}\right)=\gamma(F)+1$.

Now we are ready to present our main result.

Theorem 3. Let $G=(V, E)$ be a graph without isolated vertices and $\Gamma_{G}=(E, \gamma)$ be the independent set game on $G$. Then $\Gamma_{G}$ is convex if and only if every non-pendant edge is incident to a pendant edge in $G$. 
Proof. We first prove the "only if" part. Assume to the contrary that there is a non-pendant edge $e \in E$ which is not incident to any pendant edge in $G$. Let $u_{1}, u_{2}$ be the endpoints of $e$. Thus $d_{G}(v) \geq 2$ for any $v \in N_{G}\left(u_{1}\right) \cup N_{G}\left(u_{2}\right)$. Let $W_{u_{1} u_{2}}$ be the set of vertices from $N_{G}\left(u_{1}\right) \cap N_{G}\left(u_{2}\right)$ with degree two, i.e., $W_{u_{1} u_{2}}=\left\{w \in N_{G}\left(u_{1}\right) \cap N_{G}\left(u_{2}\right): d_{G}(w)=2\right\}$. In the following, we distinguish two cases of $W_{u_{1} u_{2}}$ and show that either case leads to a contradiction. Hence we conclude that every non-pendant edge is incident to a pendant edge in $G$.

Case 1: $\left|W_{u_{1} u_{2}}\right| \leq 1$. Let $S=\delta_{G}\left(u_{1}\right)$ and $T=\delta_{G}\left(u_{2}\right)$. Since $d_{G}(v) \geq 2$ for any $v \in N_{G}\left(u_{1}\right) \cup$ $N_{G}\left(u_{2}\right)$, we have $\gamma(S)=\gamma(T)=\alpha\left(K_{1}\right)=1$ and $\gamma(S \cap T)=\gamma(\{e\})=\alpha\left(K_{0}\right)=0$. Moreover, $\gamma(S \cup T)=\alpha\left(K_{2}\right)=1$ if $\left|W_{u_{1} u_{2}}\right|=0$, and $\gamma(S \cup T)=\alpha\left(K_{3}\right)=1$ if $\left|W_{u_{1} u_{2}}\right|=1$. It follows that $\gamma(S)+\gamma(T)>\gamma(S \cap T)+\gamma(S \cup T)$, which contradicts the convexity of $\Gamma_{G}$.

Case 2: $\left|W_{u_{1} u_{2}}\right| \geq 2$. Let $w^{*} \in W_{u_{1} u_{2}}$. Clearly, $N_{G}\left(w^{*}\right)=\left\{u_{1}, u_{2}\right\}$. The following arguments hold for $i=1,2$. Since $d_{G}(v) \geq 2$ for any $v \in N_{G}\left(u_{i}\right) \cup N_{G}\left(w^{*}\right),\left\{u_{i}, w^{*}\right\}$ is neither a pendant edge nor incident to any pendant edge. Let $W_{u_{i} w^{*}}$ be the vertex set defined analogously to $W_{u_{1} u_{2}}$, i.e., $W_{u_{i} w^{*}}=\left\{w \in N_{G}\left(u_{i}\right) \cap N_{G}\left(w^{*}\right): d_{G}(w)=2\right\}$. Instead of $W_{u_{1} u_{2}}$, we turn to consider the cardinality of $W_{u_{i} w^{*}}$. Clearly, $W_{u_{i} w^{*}} \subseteq N_{G}\left(w^{*}\right)$. It follows that $W_{u_{i} w^{*}} \subseteq\left\{u_{1}, u_{2}\right\}$. However, $\left|W_{u_{1} u_{2}}\right| \geq 2$ implies that $d_{G}\left(u_{i}\right) \geq 3$. It follows that $W_{u_{i} w^{*}}=\emptyset$ which boils down to Case 1 .

Now we prove the "if" part. Assume to the contrary that $\Gamma_{G}$ is not convex. Then there is an edge $e \in E$ and two edge sets $S \subseteq T \subseteq E \backslash\{e\}$ such that

$$
\gamma\left(S^{\prime}\right)-\gamma(S)>\gamma\left(T^{\prime}\right)-\gamma(T)
$$

where $S^{\prime}=S \cup\{e\}$ and $T^{\prime}=T \cup\{e\}$. Let $u_{1}, u_{2}$ be the endpoints of $e$. By Lemma 1, $e$ is not a pendant edge in $G$, since otherwise $\gamma\left(S^{\prime}\right)-\gamma(S)=\gamma\left(T^{\prime}\right)-\gamma(T)=1$. Moreover, Lemma 2 implies that

$$
\gamma\left(S^{\prime}\right)=\gamma(S)+1
$$

and

$$
\gamma\left(T^{\prime}\right)=\gamma(T)
$$

Let $I_{S^{\prime}}$ and $I_{T}$ be a maximum independent set in $G\left[V\left\langle S^{\prime}\right\rangle\right]$ and $G[V\langle T\rangle]$, respectively. By Lemma 2, (4) implies that an endpoint of $e$, say $u_{1}$, belongs to $I_{S^{\prime}}$ but is not adjacent to any pendant vertex. It follows that $\delta_{G}\left(u_{1}\right) \subseteq S^{\prime} \subseteq T^{\prime}$. Since $u_{1} \notin V\langle T\rangle$, we have $u_{1} \notin I_{T}$. In the following, we construct an independent set in $G\left[V\left\langle T^{\prime}\right\rangle\right]$ with $u_{1}$ from $I_{T}$, the size of which is larger than $I_{T}$. However, this contradicts (5). Hence we conclude that $\Gamma_{G}$ is convex.

It remains to construct an asserted independent set in $G\left[V\left\langle T^{\prime}\right\rangle\right]$. We first show that $I_{T} \cap$ $N_{G}\left(u_{1}\right) \neq \emptyset$ and every vertex in $I_{T} \cap N_{G}\left(u_{1}\right)$ is adjacent to a pendant vertex from $V\langle T\rangle$. Notice that $I_{T}$ is also an independent set in $G\left[V\left\langle T^{\prime}\right\rangle\right]$. If $I_{T} \cap N_{G}\left(u_{1}\right)=\emptyset$, i.e., $u_{1}$ is not adjacent to any 
vertex in $I_{T}$, then $I_{T} \cup\left\{u_{1}\right\}$ is an independent set in $G\left[V\left\langle T^{\prime}\right\rangle\right]$ with cardinality $\left|I_{T}\right|+1$, which is trivial. Hence $I_{T} \cap N_{G}\left(u_{1}\right) \neq \emptyset$. Since $u_{1}$ is not adjacent to any pendant vertex, every vertex in $I_{T} \cap N_{G}\left(u_{1}\right)$ is adjacent to a pendant vertex. Notice that $I_{T} \cap N_{G}\left(u_{1}\right) \subseteq V\langle T\rangle$. Hence every pendant vertex which is adjacent to a vertex in $I_{T} \cap N_{G}\left(u_{1}\right)$ also belongs to $V\langle T\rangle$. Now we construct a larger independent set in $G\left[V\left\langle T^{\prime}\right\rangle\right]$ with $u_{1}$ from $I_{T}$. Let $I_{T}^{\prime}$ be the vertex set obtained from $I_{T}$ by replacing each vertex in $I_{T} \cap N_{G}\left(u_{1}\right)$ with a pendant vertex from $V\langle T\rangle$ adjacent to it. Clearly, $I_{T}^{\prime} \subseteq V\langle T\rangle$. Moreover, $I_{T}^{\prime}$ is a maximum independent set in $G[V\langle T\rangle]$. Hence $I_{T}^{\prime}$ is also an independent set in $G\left[V\left\langle T^{\prime}\right\rangle\right]$. Since $u_{1}$ is not adjacent to any vertex in $I_{T}^{\prime}, I_{T}^{\prime} \cup\left\{u_{1}\right\}$ is an independent set in $G\left[V\left\langle T^{\prime}\right\rangle\right]$ with cardinality $\left|I_{T}\right|+1$.

Theorem 3 can be strengthened to a characterization for the additivity of independent set games.

Corollary 4. Let $G=(V, E)$ be a graph without isolated vertices and $\Gamma_{G}=(E, \gamma)$ be the independent set game on $G$. Then $\Gamma_{G}$ is additive if and only if every non-pendant vertex is adjacent to a pendant vertex in $G$.

Proof. Notice that if every non-pendant vertex is adjacent to a pendant vertex, then every nonpendant edge is incident to a pendant edge. Hence to prove the additivity of $\Gamma_{G}$, it suffices to show that $\Gamma_{G}$ is concave if and only if every non-pendant vertex is adjacent to a pendant vertex in $G$.

We first prove the "only if" part. Assume to the contrary that there is a non-pendant vertex $v^{*} \in V$ which is not adjacent to any pendant vertex in $G$. Let $S, T \subseteq \delta_{G}\left(v^{*}\right)$ be two non-empty edge sets such that $S \cap T=\emptyset$ and $S \cup T=\delta_{G}\left(v^{*}\right)$. It follows that $\gamma(S)=\gamma(T)=\alpha\left(K_{0}\right)=0$, $\gamma(S \cap T)=\gamma(\emptyset)=0$ and $\gamma(S \cup T)=\alpha\left(K_{1}\right)=1$. Therefore, we have $\gamma(S)+\gamma(T)<\gamma(S \cap T)+\gamma(S \cup T)$, which contradicts the concavity of $\Gamma_{G}$.

Now we prove the "if" part. Assume to the contrary that $\Gamma_{G}$ is not concave. Then there is an edge $e$ and two edge sets $S \subseteq T \subseteq E \backslash\{e\}$ such that

$$
\gamma\left(S^{\prime}\right)-\gamma(S)<\gamma\left(T^{\prime}\right)-\gamma(T)
$$

where $S^{\prime}=S \cup\{e\}$ and $T^{\prime}=T \cup\{e\}$. By Lemma 1, $e$ is not a pendant edge in $G$, since otherwise $\gamma\left(S^{\prime}\right)-\gamma(S)=\gamma\left(T^{\prime}\right)-\gamma(T)=1$. By Lemma 2, we have

$$
\gamma\left(T^{\prime}\right)=\gamma(T)+1
$$

and

$$
\gamma\left(S^{\prime}\right)=\gamma(S)
$$

Moreover, (7) implies that an endpoint of $e$ is neither a pendant vertex nor adjacent to any pendant vertex, which contradicts our basic assumption. 
One natural question is that whether there are graphs inducing convex but not additive independent set games. In Figure 1, we enumerate all connected graphs with 5 vertices that induce convex independent set games. Clearly, $G_{1}$ and $G_{2}$ also induce additive independent set games, but $G_{3}$ and $G_{4}$ do not induce additive independent set games.

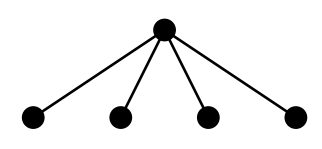

$G_{1}$

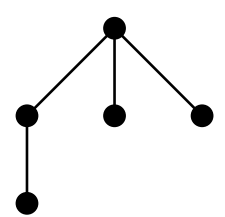

$G_{2}$

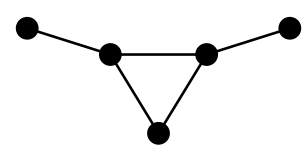

$G_{3}$

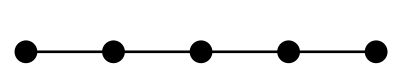

$G_{4}$

Figure 1: All connected graphs with 5 vertices that induce convex independent set games.

Since pendant edges and pendant vertices can be recognized in polynomial time by checking the degree of every vertex, Theorem 3 and Corollary 4 suggest that both convex instances and additive instances of independent set games can be recognized efficiently.

Corollary 5. Let $G=(V, E)$ be a graph without isolated vertices and $\Gamma_{G}=(E, \gamma)$ be the independent set game on $G$. Then both convexity and additivity of $\Gamma_{G}$ can be determined in polynomial time.

\section{Convexity of relaxed independent set games}

Independent set games introduced by Deng et al. [5] have limitation in the sense that every project requires all suitable participants to cooperate to be done. Hence a project fails if it misses any suitable participant in a coalition. However, a project might only require parts of suitable participants to cooperate to be done, not necessarily all suitable participants. Here we consider a special case of this scenario, where every project requires all suitable participants in the current coalition, not necessarily all suitable participants in the grand coalition, to cooperate to be done. Independent set games complying with the new requirement are called relaxed independent set games. Formally, the relaxed independent set game is a cooperative game $\hat{\Gamma}_{G}=(E, \hat{\gamma})$ defined on a graph $G=(V, E)$, where $E$ is the set of players and $\hat{\gamma}: 2^{E} \rightarrow \mathbb{N}$ is the characteristic function such that $\hat{\gamma}(F)=\alpha(G[F])$ for any $F \subseteq E$. We show that the convexity of relaxed independent set games admits an efficient characterization.

Theorem 6. Let $G=(V, E)$ be a graph without isolated vertices and $\hat{\Gamma}_{G}=(E, \hat{\gamma})$ be the relaxed independent set game on $G$. Then $\hat{\Gamma}_{G}$ is convex if and only if $G$ is $\left(K_{3}, P_{4}\right)$-free. 
Proof. We first prove the "only if" part. Assume to the contrary that $G$ is not $\left(K_{3}, P_{4}\right)$-free. We show that any subgraph isomorphic to $K_{3}$ or $P_{4}$ gives rise to non-convex instances of $\hat{\Gamma}_{G}$.

Assume that $H$ is either $K_{3}$ or $P_{4}$. Let $S \subseteq E(H)$ be a set consisting of two incident edges in $H$, and let $T=E(H) \backslash S$. Notice that $\hat{\gamma}(S)=\alpha\left(P_{3}\right)=2, \hat{\gamma}(T)=\alpha\left(K_{2}\right)=1$, and $\hat{\gamma}(S \cap T)=\hat{\gamma}(\emptyset)=0$. Moreover, $\hat{\gamma}(S \cup T)=\alpha\left(K_{3}\right)=1$ if $H=K_{3}$ and $\hat{\gamma}(S \cup T)=\alpha\left(P_{4}\right)=2$ if $H=P_{4}$. In either case, we have $\hat{\gamma}(S)+\hat{\gamma}(T)>\hat{\gamma}(S \cap T)+\hat{\gamma}(S \cup T)$, which contradicts the convexity of $\hat{\Gamma}_{G}$.

Now we prove the "if" part. Let $H_{1}, \ldots, H_{r}$ be the components of $G$. Okamoto [15] showed that a graph is $\left(K_{3}, P_{4}\right)$-free if and only if every component is a star. It follows that

$$
\alpha(G)=\sum_{i=1}^{r} \alpha\left(H_{i}\right)=\sum_{i=1}^{r}\left|E\left(H_{i}\right)\right|=|E| .
$$

Furthermore, we have

$$
\hat{\gamma}(F)=\alpha(G[F])=\sum_{i=1}^{r}\left|E\left(H_{i}\right) \cap F\right|=|F|
$$

for any $F \subseteq E$. Let $e \in E$ and $S \subseteq T \subseteq E \backslash\{e\}$. It follows that

$$
\hat{\gamma}\left(S^{\prime}\right)-\hat{\gamma}(S)=\left|S^{\prime}\right|-|S|=1
$$

and

$$
\hat{\gamma}\left(T^{\prime}\right)-\hat{\gamma}(T)=\left|T^{\prime}\right|-|T|=1,
$$

where $S^{\prime}=S \cup\{e\}$ and $T^{\prime}=T \cup\{e\}$. Therefore, $\hat{\Gamma}_{G}$ is additive and hence convex.

From the proof above, Theorem 6 can be strengthened as follows.

Corollary 7. Let $G=(V, E)$ be a graph without isolated vertices and $\hat{\Gamma}_{G}=(E, \hat{\gamma})$ be the relaxed independent set game on $G$. Then $\hat{\Gamma}_{G}$ is additive if and only if $G$ is $\left(K_{3}, P_{4}\right)$-free.

Since $\left(K_{3}, P_{4}\right)$-free graphs can be recognized in polynomial time, Theorem 6 and Corollary 7 suggest that convex (actually additive) instances of relaxed independent set games can be recognized efficiently.

Corollary 8. Let $G=(V, E)$ be a graph without isolated vertices and $\hat{\Gamma}_{G}=(E, \hat{\gamma})$ be the relaxed independent set game on $G$. Then convexity (actually additivity) of $\hat{\Gamma}_{G}$ can be determined in polynomial time. 


\section{Concluding remarks}

We study the convexity of independent set games in this paper. We show that an independent set game is convex if and only if every non-pendant edge is incident to a pendant edge in the underlying graph. Since convex games are population monotonic and hence totally balanced, a possible direction for future work is to characterize population monotonicity or total balancedness of independent set games based on the characterization of convexity. Deng et al. [5] proved that an independent set game is balanced if and only if the underlying graph is a König-Egerváry graph. Thus any characterization for convexity, population monotonicity or total balancedness of independent set games also provides a sufficient condition for König-Egerváry graphs.

\section{Acknowledgments}

The authors are grateful to anonymous referees for valuable comments and helpful suggests that greatly improved the presentation of this paper.

\section{References}

[1] M. J. Albizuri and H. Hamers. Graphs inducing totally balanced and submodular Chinese postman games. Discrete Applied Mathematics, 172:98-103, 2014.

[2] R. J. Aumann and M. Maschler. Game theoretic analysis of a bankruptcy problem from the Talmud. Journal of Economic Theory, 36(2):195-213, 1985.

[3] I. Curiel, G. Pederzoli, and S. Tijs. Sequencing games. European Journal of Operational Research, 40(3):344-351, 1989.

[4] R. W. Deming. Independence numbers of graphs - an extension of the Koenig-Egervary theorem. Discrete Mathematics, 27(1):23-33, 1979.

[5] X. Deng, T. Ibaraki, and H. Nagamochi. Algorithmic aspects of the core of combinatorial optimization games. Mathematics of Operations Research, 24(3):751-766, 1999.

[6] M. Grötschel, L. Lovász, and A. Schrijver. Geometric Algorithms and Combinatorial Optimization. Springer, 1993.

[7] H. Hamers. On the concavity of delivery games. European Journal of Operational Research, 99(2):445-458, 1997. 
[8] H. Hamers, P. Borm, and S. Tijs. On games corresponding to sequencing situations with ready times. Mathematical Programming, 69(1-3):471-483, 1995.

[9] Y. T. Herer and M. Penn. Characterizations of naturally submodular graphs: a polynomially solvable class of the TSP. Proceedings of the American Mathematical Society, 123(3):673-679, 1995.

[10] M. Kobayashi and Y. Okamoto. Submodularity of minimum-cost spanning tree games. Networks, 63(3):231-238, 2014.

[11] Z. K. Koh and L. Sanità. An efficient characterization of submodular spanning tree games. Mathematical Programming, 183:359-377, 2020.

[12] J. Kuipers. A polynomial time algorithm for computing the nucleolus of convex games. Reports in operations research and systems theory. Maastricht University, 1996.

[13] S. C. Littlechild and G. Owen. A simple expression for the Shapley value in a special case. Management Science, 20(3):370-372, 1973.

[14] M. Maschler, B. Peleg, and L. S. Shapley. The kernel and bargaining set for convex games. International Journal of Game Theory, 1(1):73-93, 1971.

[15] Y. Okamoto. Submodularity of some classes of the combinatorial optimization games. Mathematical Methods of Operations Research, 58(1):131-139, 2003.

[16] T. T. Platz. On the submodularity of multi-depot traveling salesman games. Discrete Applied Mathematics, 255:75-85, 2019.

[17] A. Schrijver. Combinatorial Optimization: Polyhedra and Efficiency. Number 24 in Algorithms and Combinatorics. Springer, 2003.

[18] L. S. Shapley. Cores of convex games. International Journal of Game Theory, 1(1):11-26, 1971.

[19] M. Shubik. Game theory models and methods in political economy. Handbook of Mathematical Economics, 1:285-330, 1981.

[20] A. van den Nouweland and P. Borm. On the convexity of communication games. International Journal of Game Theory, 19(4):421-430, 1991. 